\title{
Pancreatite aguda grave por hipertrigliceridemia em um hospital terciário em Recife-PE
}

\section{Serious acute pancreatitis by hypertriglyceridemia at a terciary hospital in Recife-PE}

Maria Laryssa da Silva Pontes ${ }^{1}$, Evelliny Gomes da Silva ${ }^{2}$, Bruno Vinicius Nascimento da Silva ${ }^{3}$, Phablo Fernandes José Oliveira Ferreira ${ }^{3}$.

${ }^{1}$ Residente de Clínica Médica do Hospital Getulio Vargas, Recife, Pernambuco, Brasil

${ }^{2}$ Universidade Federal de Pernambuco, Campos Acadêmico do Agreste, Recife, Brasil

${ }^{3}$ Universidade Federal de Pernambuco, Recife. Brasil

\section{Resumo}

A hipertrigliceridemia (HTG) é uma das causas de pancreatite aguda, sendo responsável por até $10 \%$ de todos os casos, podendo ser potencialmente fatal quando associada a uma hipertrigliceridemia grave. Nenhuma diretriz de tratamento para pancreatite aguda por hipertrigliceridemia (HTG-PA) foi estabelecida, embora tratamentos eficazes como insulina, heparina, plasmaférese e medicamentos antiHTG tenham sido implementados em pacientes com HTG-PA. Este trabalho objetiva discutir e descrever a abordagem e condução de um caso de HGT-PA em um Hospital Terciário do SUS na cidade de RecifePE.

Palavras-chaves: Pancreatite aguda, hipertrigliceridemia, necrose pancreática

\begin{abstract}
Hypertriglyceridemia (HTG) is one of the causes of acute pancreatitis, accounting for up to $10 \%$ of all cases, and can be potentially fatal when associated with severe hypertriglyceridemia. No treatment guidelines for acute pancreatitis due to hypertriglyceridemia (HTG-AP) have been established, although effective treatments such as insulin, heparin, plasmapheresis and anti-HTG drugs have been implemented in patients with HTG-AP. This work has as objective to discuss and describe the approach and management of a case of HGT-AP in a Tertiary Hospital of SUS in the city of Recife-PE.

Keywords: Acute pancreatitis, hypertriglyceridemia, pancreatic necrosis
\end{abstract}

\section{Introdução}

A pancreatite aguda por hipertrigliceridemia grave pode ser potencialmente fatal e hoje é um dos principais casos de Pancreatite Aguda (PA) no mundo ${ }^{1}$. O curso clínico da pancreatite induzida por hipertrigliceridemia (HTG-PA) é altamente semelhante ao da PA de outras etiologias, como a etílica ${ }^{2}$. Destaca-se que a HGTP pode está associada a erros inatos do metabolismo, como deficiência de lipoproteína lipase, deficiência de apoproteína C-II e hipertrigliceridemia familiar e geralmente, seu curso clínico é descrito por altos níveis plasmáticos de triglicerídeos (> $1000 \mathrm{mg} / \mathrm{dL}$ ), restos de quilomícrons ou partículas semelhantes a lipoproteínas de densidade intermediária e / ou quilomícrons ${ }^{1}$. 
É importante descrever também que no momento, nenhuma diretriz de tratamento para HTG-AP foi estabelecida, embora tratamentos eficazes como insulina, heparina, plasmaférese e medicamentos anti-HTG tenham sido implementados em pacientes com HTG-AP ${ }^{3}$.

Com efeito, este trabalho tem como objetivo discutir e descrever sobre a abordagem e condução de um caso de HGT-PA em um Hospital Terciário do SUS na cidade de Recife-PE, a partir da busca de prontuário.

\section{Relato de caso}

Paciente 34 anos, com quadro prévio há 4 anos de PA e histórico de Colecistectomia há 6 anos, deu entrada na emergência do HGV com dor abdominal em epigástrio irradiando para hipocôndrio direito e dorso, associado a presença de náuseas e vômitos. No município de procedência, realizou exames laboratoriais que evidenciaram amilase e lipase aumentadas, sendo então aventada a hipótese de PA. Desse modo, a paciente foi encaminhada a serviço terciário de emergência.

\section{Como a paciente apresentava-se} clinicamente estável foi encaminhada a enfermaria de Clínica Médica do Hospital Getulio Vargas, onde fora, solicitado novos exames (descritos no quadro 01), inclusive perfil lipídico em que foi evidenciado hipertrigliceridemia (6925 mg/dL) e solicitado USG de abdome total (descrita no quadro 01), fechando critérios para HGT-PA. Foi realizada estratificação da paciente (não grave) sendo iniciamos uma infusão intravenosa (IV) de insulina regular a uma taxa de 0,1 a 0,3 unidades / $\mathrm{kg} /$ hora, enquanto monitoriza de perto os níveis de glicose no sangue. Optamos por 0,1 unidade $/ \mathrm{kg} /$ hora, sendo iniciado 8UI. Nesse contexto, a paciente evoluiu com melhora do quadro, havendo queda importante da hipertrigliceridemia para $525 \mathrm{mg} / \mathrm{dL}$ em 3 dias de terapêutica.

Porém, após 2 semanas, evoluiu com piora da dor abdominal associado a presença de sinais de Síndrome da Resposta Inflamatória Sistêmica (SIRS), sendo realizada TC de abdome com contraste que evidenciou pequenas áreas de necrose e coleções peripancreáticas bilaterais com necrose inferior a $30 \%$ (descritas no quadro 01), além da presença de coleção extensa (Balthazar: 6 pontos). Diante do quadro foi iniciado antibioticoterapia com carbapenêmico (imipeném) por 10 dias, evoluindo com melhora clínica e recebendo alta hospitalar do serviço para acompanhamento ambulatorial.

Quadro 01: evolução dos exames laboratoriais e de imagem da paciente.

\begin{tabular}{|c|c|}
\hline Exame & Resultado \\
\hline $\begin{array}{l}\text { Laboratório } \\
\text { da admissão } \\
\text { no HGV }\end{array}$ & $\begin{array}{l}\text { Hemácias: 3,89/ Hb: 13,3/ VCM: 94,6/ } \\
\text { HCM: 34,2/ CHCM: 36,1/ Ht: 36,8/ RDW: } \\
\text { 10,8/ TGP: 15/ U: 28/ Cr: 1,1/ Na: 135/ Mg: } \\
\text { 2/ K: 4/ Leuco 16.950/ Bast: 0/ Seg: 35,1\%/ } \\
\text { Eos:6,6\%/ Linf: 31,1\%/ PLQ: 176.000/ } \\
\text { COLEST TOTAL: 325/ TG: 6.925/ } \\
\text { Amilase:145/Lipase: 283/ FA: } 60\end{array}$ \\
\hline $\begin{array}{ll}\text { USG } & \text { da } \\
\text { admissão } & \text { no } \\
\text { HGV } & \end{array}$ & $\begin{array}{l}\text { Fígado apresenta dimensões, topografia, } \\
\text { forma e contornos normais. A ecotextura é } \\
\text { homogênea com arquitetura vascular } \\
\text { preservada. Apresenta aumento difuso da } \\
\text { ecogenicidade do parênquima com } \\
\text { atenuação sonora posterior, sugerindo } \\
\text { esteatose moderada. Vias biliares intra e } \\
\text { extra-hepáticas de trajeto e calibre } \\
\text { conservados. Vesícula biliar ausente } \\
\text { (cirurgia prévia). Pâncreas de forma e } \\
\text { volume, notando-se }\end{array}$ \\
\hline
\end{tabular}




\begin{tabular}{|c|c|}
\hline & $\begin{array}{l}\text { hipoecogenicidade em topografia da cabeça. } \\
\text { Presença de alças sem peristalse adjacente a } \\
\text { cabeça pancreática, associada a área } \\
\text { hipoecoica mal definida, medindo } 7,6 \text { x } 5,5 \\
\text { cm. Adjacente ao polo inferior do rim } \\
\text { direito, identifica-se imagem hipoecoica, } \\
\text { bem delimitada medindo } 8,2 \times 7,9 \text { x } 4,1 \mathrm{~cm} \\
\text { (coleção?). Aparentemente se continua com } \\
\text { imagem acima descrita. Há imagem em } \\
\text { "pseudorrim", fixa, nessa região, sugerindo } \\
\text { espessamento parietal de alça intestinal. } \\
\text { Nota-se fina lâmina líquida em espaço } \\
\text { hepatorrenal. Baço de forma, volume e } \\
\text { contornos normais. Rins tópicos, de forma, } \\
\text { contornos e dimensões normais. Relação } \\
\text { parênquima/seio renal conservada. Ausência } \\
\text { de massas císticas ou sólidas, sinais de } \\
\text { hidronefrose, coleções líquidas } \\
\text { perinefréticas ou imagem compatível com } \\
\text { litíase, detectável pelo método. Aorta } \\
\text { abdominal e veia cava inferior de trajeto e } \\
\text { calibre conservados, com paredes regulares. } \\
\text { Bexiga vazia. }\end{array}$ \\
\hline $\begin{array}{ll}\text { TC } & \text { da } \\
\text { admissão } & \text { no } \\
\text { HGV } & \end{array}$ & $\begin{array}{l}\text { Opacidades em vidro fosco em ambas as } \\
\text { bases pulmonares. Fígado de topografia, } \\
\text { volume e contornos preservados sem sinais } \\
\text { de lesões expansivas ou dilatação das vias } \\
\text { biliares intra-hepáticas. Leve hipoatenuação } \\
\text { difusa do parênquima hepático, inferindo } \\
\text { esteatose. Pequeno granuloma calcificado } \\
\text { no segmento VII. Vesícula biliar não } \\
\text { caracterizada. Pâncreas apresentando } \\
\text { aumento volumétrico da cabeça e processo } \\
\text { uncinado. Observa-se obliteração e líquido } \\
\text { na gordura peripancreática adjacente, além } \\
\text { de importante quantidade de líquido livre } \\
\text { nos espaços pararrenais, estendendo-se pela } \\
\text { goteira parietocólica direita até a escavação } \\
\text { pélvica, sem coleções organizadas. O } \\
\text { conjunto dos achados é compatível com } \\
\text { pancreatite aguda. Baço de aspecto } \\
\text { anatômico. Rins de forma, dimensões, } \\
\text { contorno e atenuação normais, eliminando } \\
\text { satisfatoriamente o meio de contraste. } \\
\text { Adrenais visíveis, sem alterações. Aorta e } \\
\text { veia cava inferior com dimensões e } \\
\text { contornos normais. Distribuição habitual de } \\
\text { alças delgadas e cólicas. Bexiga de } \\
\text { morfologia e capacidade preservadas, com } \\
\text { paredes finas e regulares, sem evidência de } \\
\text { falhas de enchimento. Útero de morfologia, } \\
\text { volume e valores de atenuação dentro da } \\
\text { normalidade. Não detectamos } \\
\text { linfonodomegalias. Flebólitos na escavação } \\
\text { pélvica. }\end{array}$ \\
\hline $\begin{array}{lr}\text { TC após } 2 \\
\text { semanas da }\end{array}$ & $\begin{array}{l}\text { Pâncreas de espessura relativamente } \\
\text { preservada, entretanto com relativo aumento }\end{array}$ \\
\hline
\end{tabular}

\begin{tabular}{|c|c|}
\hline admissão & $\begin{array}{l}\text { nas dimensões da sua cabeça, com leve } \\
\text { irregularidade em seus contornos e } \\
\text { pequenas áreas de necrose, mais evidentes } \\
\text { na periferia do processo unciforme e cabeça } \\
\text { do pâncreas. Associam-se coleções } \\
\text { peripancreáticas, com paredes bem } \\
\text { definidas, netse estudo, que se estendem às } \\
\text { regiões pré-fasciais renais, retrocavidade } \\
\text { dos epíplons, ângulo esplênico e às goteiras } \\
\text { parietocólicas bilateralmente, associados a } \\
\text { aumento no número de linfonodos. Parece } \\
\text { existir comunicação entre as coleções, com } \\
\text { a espessura média das mesmas de até } 4 \text { cm, } \\
\text { estendendo-se longitudinalmente por até } 12 \\
\text { cm à direita e } 20 \text { cm à esquerda. O quadro é } \\
\text { compatível com pancreatite subaguda } \\
\text { complicada por coleções peripancreáticas } \\
\text { com necrose inferior a } 30 \% \text { (índice de } \\
\text { severidade por CT: } 6 \text { pontos). }\end{array}$ \\
\hline
\end{tabular}

\section{Discussão}

A hipertrigliceridemia (HTG) é uma das principais causas de pancreatite (PA), sendo responsável por até $10 \%$ de todos os $\operatorname{casos}^{4,5}$. O risco aumenta progressivamente com níveis séricos de triglicerídeos acima de $500 \mathrm{mg} / \mathrm{dL}$, e o risco de desenvolver pancreatite aguda é de aproximadamente $5 \%$ com triglicerídeos séricos> $1000 \mathrm{mg} / \mathrm{dL}$ e 10 a $20 \%$ com triglicerídeos $>2000$ $\mathrm{mg} / \mathrm{Dl}^{6}$. No caso descrito, a paciente apresentava risco expressivo, uma vez que, à admissão, tinha uma HTG de $6925 \mathrm{mg} / \mathrm{dL}$. Embora a maioria dos casos seja bastante leve, existe um subconjunto de pacientes com pancreatite grave em que a mortalidade pode atingir até $30 \%{ }^{4}$. Existem estudos bem conduzidos sugerindo que o HTG-AP está associado a uma maior taxa de gravidade e complicações, conforme evidenciado no caso $^{2,4}$.

Embora o mecanismo preciso pelo qual o HTG cause PA não seja totalmente compreendido, acredita-se que tanto a HTG (causando excesso de ácidos graxos livres) quanto os quilomícrons 
aumentem a viscosidade plasmática, o que pode induzir isquemia no tecido pancreático e desencadear inflamação de órgãos ${ }^{5}$. A HTG pode ser primária (associada a distúrbios genéticos) ou secundária (associada a medicamentos, gravidez, diabetes mellitus ou álcool, por exemplo). O curso clínico da pancreatite induzida por HTG (HTGP) é altamente semelhante ao da PA de outras etiologias, sendo o HTG a única característica clínica distintiva $^{2}$. A etiologia da HTG da paciente não foi encontrada na internação, sendo objetivo das consultas ambulatoriais de acompanhamento, mas acredita-se que possa estar associada a uma causa primária.

As medidas terapêuticas no HTG-AP incluem modificações na dieta, diferentes agentes anti-hiperlipidêmicos, tratamento com insulina e / ou heparina. O uso benéfico da plasmaférese é relatado e sugerido repetidamente em muitos estudos, principalmente em pacientes com uma ou mais características preocupantes (como acidose lática ou SIRS) $)^{4,7}$. Todavia, lembra-se que o tratamento inicial de um paciente com PA consiste em cuidados de suporte com reanimação fluida, controle da dor e suporte nutricional.

A insulina promove o metabolismo dos ácidos graxos e reduz os níveis de triglicerídeos, inclusive, regimes de insulina reduzem os níveis de triglicerídeos para menos de $500 \mathrm{mg} / \mathrm{dL}$ durante 3,5 a $4 \operatorname{dias}^{8,9}$. Indica-se a infusão intravenosa (IV) de insulina regular a uma taxa de 0,1 a 0,3 unidades/kg/hora, com monitorização glicêmica periódica e, em pacientes com níveis de glicose séricos entre 150 e $200 \mathrm{mg} / \mathrm{dL}$, deve-se adicionar uma infusão separada de 5\% de dextrose para evitar hipoglicemia ${ }^{8}$.

\section{Conclusão}

A pancreatite por hipertrigliceridemia é uma causa mais rara, porém importante, de pancreatite aguda que deve ser prontamente identificada e tratada o mais rápido possível. Portanto, o conhecimento desta afecção pelo médico generalista é de grande importância e o tratamento sugerido é simples, acessível e apresenta boa resposta clínica, conforme descrito e discutido neste trabalho.

\section{Referências}

1. Stefanutti C, Labbadia G, Morozzi C. Severe (2013) Hypertriglyceridemia-Related Acute Pancreatitis, Therapeutic Apheresis and Dialysis, 17: 130-7.

2. Rawla P, Sunkara T, Thandra KC, Gaduputi V. (2018) Hypertriglyceridemia-induced pancreatitis: updated review of current treatment and preventive strategies, Clinical Journal of Gastroenterology, 11: 441-8.

3. Yao-Yao Guo, Hui-Xia Li, Yue Zhang, Wen-Hua He. (2019) Hypertriglyceridemia-induced acute pancreatitis: progress on disease mechanisms and treatment modalities, Discov Med, 101-9.

4. Valdivielso P, Ramírez-Bueno A, Ewald N. (2014) Current knowledge of hypertriglyceridemic pancreatitis, European Journal of Internal Medicine 25:689-94.

5. de Pretis N, Amodio A, Frulloni L. (2018) Hypertriglyceridemic pancreatitis: Epidemiology, pathophysiology and clinical management, United European Gastroenterology Journal, 6: 649-55.

6. Scherer J, Singh VP, Pitchumoni CS, Yadav D. (2014) Issues in hypertriglyceridemic pancreatitis: An update, J Clin Gastroenterol, 48:195-203.

7. Ipe TS, Pham HP, Williams LA. (2018) Critical updates in the 7th edition of the American Society for Apheresis guidelines, Journal of 
Clinical Apheresis, 33:78-94.

8. Mikhail N, Trivedi K, Page C, Wali S, Cope D. (2005) Treatment of severe hypertriglyceridemia in nondiabetic patients with insulin, Am J Emerg Med, 23:415-7.

9. Jabbar MA, Zuhri-Yafi MI, Larrea J. (1998) Insulin therapy for a non-diabetic patient with severe hypertriglyceridemia, J Am Coll Nutr, 17(5):458-61.
Autor para correspondência

Maria Laryssa da Silva Pontes dralarypontes@gmail.com

Recebido: 23 de agosto de 2020 Aceito: 14 de dezembro de 2020 